\title{
FIRST HITTING TIMES OF SIMPLE RANDOM WALKS ON GRAPHS WITH CONGESTION POINTS
}

\author{
MIHYUN KANG
}

Received 29 July 2002

\begin{abstract}
We derive the explicit formulas of the probability generating functions of the first hitting times of simple random walks on graphs with congestion points using group representations.
\end{abstract}

2000 Mathematics Subject Classification: 60G50, 60B15, 60K30, 05A15.

1. Introduction. Random walk on a graph is a Markov chain whose state space is the vertex set of the graph and whose transition from a given vertex to an adjacent vertex along an edge is defined according to some probability distribution. The probability distribution might depend on vertices, and the case of the uniform distribution over incident edges is called a simple random walk. Many researches have been done on various aspects of random walks such as transience or recurrence, asymptotic behavior of transition probabilities, convergence rates to its stationary distributions, and convergence to a boundary and harmonic functions $[2,6]$.

Random walks can describe the structure of graphs, groups, and related objects and the structure of computer networks or electric networks [3, 8]. It is quite useful to devise probabilistic algorithms of random walks on graphs which reflect combinatorial problems when deterministic methods to analyze them are known to be difficult [10]. It is well known that random walks play a crucial role in the design of randomized algorithms (off- or online) [4, 11, 15].

The first hitting time (also called the first passage time) is the time taken to reach a vertex for the first time starting from another vertex. This is one of the classical problems on Markov chains, and one can investigate the probabilistic properties of the first hitting time, the stopping time, or transition probabilities [1, 12]. The expected hitting times for random walks on graphs have been computed using the relation between electrical networks and random walks $[9,13,14]$ and for random walks on finite groups using group representations [5]. The group representation approach has been quite powerful in measuring the convergence rate of random walks to its stationary distributions [5, 7].

In this paper, we apply group representation technique for a simple random walk on finite graphs with cutvertices (or congestion points), which can be decomposed into several finite groups. Finite graphs with congestion points may 
capture the delays in computer, telecommunication, and the transportation systems, and the probability generating functions would be useful in performance evaluation of stochastic models, which arise from those various systems.

2. Preliminaries. Let $G$ be a finite group with the identity $e$. Let $V$ be a vector space of dimension $d$ over $\mathbb{C}$ of complex numbers, and let GL( $V$ ) be the group of invertible matrices of rank $d$ which represent invertible linear transformations from $V$ to $V$. A linear representation $\rho$ of $G$ is a continuous map $\rho: G \rightarrow \mathrm{GL}(V)$ satisfying $\rho(x y)=\rho(x) \rho(y)$ for every $x, y \in G$. It follows that $\rho(e)$ is the identity matrix $I$ and $\rho\left(x^{-1}\right)=\rho(x)^{-1}$. The dimension of $V$ is called the dimension of $\rho$ and is denoted by $d_{\rho}$. A linear representation $\rho$ is called a trivial representation if $\rho(x)=1$ for all $x \in G$. It is called irreducible if $V$ is not $\{0\}$ and no subspace of $V$ is stable under $G$. In other words, there is no subspace $W$ of $V$ except for $\{0\}$ and $V$ such that $\rho(x) W \subset W$ for all $x \in G$. Denote by $\hat{G}$ the set of all irreducible representations of $G$. For each $x \in G$, put $\chi_{\rho}(x)=\operatorname{Tr}(\rho(x))$, where $\operatorname{Tr}(\rho(x))=\sum_{i}(\rho(x))_{i i}$, that is, the trace of $\rho(x)$. The complex-valued function $x_{\rho}$ is called the character of $\rho$. For a finite abelian group $G$, every irreducible representation of $G$ is of dimension 1 and hence $\chi_{\rho}(x)=\rho(x)$. For a function $\mu$ defined on $G$, the Fourier transform $\hat{\mu}: \hat{G} \rightarrow \mathbb{C}$ is defined by

$$
\widehat{\mu}(\rho)=\sum_{x \in G} \mu(x) \rho(x)
$$

and the Fourier inversion formula is given by

$$
\mu(x)=\frac{1}{|G|} \sum_{\rho \in \hat{G}} d_{\rho} \operatorname{Tr}\left(\rho\left(x^{-1}\right) \hat{\mu}(\rho)\right),
$$

where $|G|$ denotes the number of elements in $G$.

Let $\mu$ be a probability distribution on $G$. Suppose that $E=\{x \in G \mid \mu(x)>0\}$ generates $G$; in other words, the set of all finite products of some elements in $E$ is equal to $G$. Then the neighbor of $x$ is given by $N_{x}=\{g x, g \in E\}$. Consider the transition matrix $P=\left(P_{x, y}\right)_{x, y \in G}$ given by

$$
P_{x, y}= \begin{cases}\mu\left(y x^{-1}\right), & \text { if } y \in N_{x} \\ 0, & \text { otherwise }\end{cases}
$$

which defines a random walk which moves from $x$ to $y$ with probability $\mu\left(y x^{-1}\right)$. Define the convolution $\mu * \mu$ by

$$
(\mu * \mu)(x)=\sum_{y \in G} \mu\left(x y^{-1}\right) \mu(y), \quad x \in G
$$


and define $\mu^{* k}=\mu^{*(k-1)} * \mu$. Then

$$
(\mu * \mu)(x)=\sum_{y \in G} P_{y, x} P_{e, y}=\sum_{y \in G} P_{e, y} P_{y, x}=\left(P^{2}\right)_{e, x},
$$

and hence $(\mu * \mu)(x)$ is the probability that a random path arrives at $x$ in two steps when it starts from $e$. Similarly, $\mu^{* k}(x)$ is the probability that the path arrives at $x$ in $k$ steps starting from $e$. In particular, a simple random walk is defined by

$$
P_{x, y}= \begin{cases}\frac{1}{\left|N_{x}\right|}, & \text { if } y \in N_{x} \\ 0, & \text { otherwise. }\end{cases}
$$

Diaconis [5] considered another random walk which starts from $x$ and is absorbed the first time it hits $S(\subset G)$ whose elements are called sinks. In other words, if a random path arrives at $S$ once, then it permanently stays there. Let $a_{k}(t)$ be the probability of arriving at $t$ at time $k$ (i.e., in $k$ steps) in the restricted random walk with $S \neq \phi$. Then $a_{k}(t)$ can be interpreted as follows:

(1) if $t \notin S$, then it is the probability of the path being at $t$ at time $k$ without having hit any sites in $S$;

(2) if $t \in S$, then it is the probability of first being absorbed at $t$ at time $k$. Let $b_{k}(t)$ be the probability of arriving at $t$ at time $k$ in the unrestricted random walk $(S=\phi)$, namely, $b_{k}(t)=\mu^{* k}\left(t x^{-1}\right)$. Let $A(t, z)$ and $B(t, z)$ be probability generating functions given by

$$
A(t, z)=\sum_{k=0}^{\infty} a_{k}(t) z^{k}, \quad B(t, z)=\sum_{k=0}^{\infty} b_{k}(t) z^{k} .
$$

FACT 2.1. (i) For $t \in S$,

$$
B(t, z)=\sum_{s \in S} A(s, z) B\left(t s^{-1} x, z\right),
$$

and for $t \notin S$,

$$
B(t, z)=A(t, z)+\sum_{s \in S} A(s, z) B\left(t s^{-1} x, z\right) .
$$

(ii) For $t \in G$,

$$
B(t, z)=\frac{1}{|G|}\left(\frac{1}{1-z}+\sum_{1 \neq \rho \in \hat{G}} d_{\rho} \operatorname{Tr}\left(\rho\left(x t^{-1}\right)[I-\hat{\mu}(\rho) z]^{-1}\right)\right) .
$$

EXAMPLE 2.2. Consider a simple random walk on $\mathbb{Z}_{n}=\{0, \ldots, n-1\}$ with a probability distribution $\mu$ such that $\mu(1)=\mu(-1)=1 / 2$. 
The dual group $\widehat{\mathbb{Z}_{n}}$ consists of characters of the form

$$
\chi_{k}(x)=\exp \left(\frac{2 \pi i k x}{n}\right), \quad x, k \in \mathbb{Z}_{n},
$$

and the Fourier transform of $\mu$ is defined by

$$
\hat{\mu}\left(\chi_{k}\right)=\cos \left(\frac{2 \pi k}{n}\right), \quad k \in \mathbb{Z}_{n}
$$

From Fact 2.1, the generating function of the first hitting time from $x$ to $t$ is

$$
U_{n}(x, t, z)=\frac{1+(1-z) \sum_{k=1}^{n-1} \cos (2 \pi k(x-t) / n)[1-\cos (2 \pi k / n) z]^{-1}}{1+(1-z) \sum_{k=1}^{n-1}[1-\cos (2 \pi k / n) z]^{-1}}
$$

and the generating function of the first hitting time from $x$ to $t$ without having hit $s \neq t$ on $\mathbb{Z}_{n}$ is

$$
\begin{aligned}
& V_{n}(x, t, s, z) \\
& =\frac{1}{n} \sum_{k=1}^{n-1}\left[\cos \left(\frac{2 \pi k(x-t)}{n}\right)-\cos \left(\frac{2 \pi k(s-t)}{n}\right)\right]\left[1-\cos \left(\frac{2 \pi k}{n}\right) z\right]^{-1} \\
& -\frac{1}{n}\left(\frac{1+(1-z) \sum_{k=1}^{n-1} \cos (2 \pi k(s-t) / n)[1-\cos (2 \pi k / n) z]^{-1}}{1+(1-z) \sum_{k=1}^{n-1}[1-\cos (2 \pi k / n) z]^{-1}}\right) \\
& \quad \times\left(\sum_{k=1}^{n-1}\left(\cos \left(\frac{2 \pi k(x-s)}{n}\right)-1\right)\left[1-\cos \left(\frac{2 \pi k}{n}\right) z\right]^{-1}\right) .
\end{aligned}
$$

For notational convenience,

$$
f(z)=V_{n}(n-2,0, n-1, z), \quad g(z)=V_{n}(n-2, n-1,0, z) .
$$

LEMMA 2.3. Consider a simple random walk on a line L, consisting of $n+1$ vertices, $\{0, \ldots, n\}$. For $x, y, t \in L$, let $T_{x, y}^{t}$ be the first hitting time from $x$ to $y$ without having hit $t$ and let $C_{x, y}^{t}(z)=\sum_{k=0}^{\infty} \operatorname{Pr}\left(T_{x, y}^{t}=k\right) z^{k}$. Then

$$
C_{n-1,0}^{n}(z)=\frac{z f(z)}{2+z g(z)}, \quad C_{1,0}^{n}(z)=-g(z)+\frac{z f(z)^{2}}{2+z g(z)}
$$

Proof. Consider a random path on $L$ from $x$ to 0 without having hit $n$. 
If $x$ is equal to $n-1$, then each path from $x$ to 0 without having hit $n$ should move the first step to $n-2$ with probability $1 / 2$. In other words,

$$
2 C_{n-1,0}^{n}(z)=z C_{n-2,0}^{n}(z) .
$$

For $1 \leq x \leq n-2$, each path from $x$ to 0 without having hit $n$ is either a path from $x$ to 0 without passing $n-1$ on the way or a path which hits $n-1$ before arriving at 0 and then restarts a random walk from $n-1$ to reach 0 without passing $n$;

$$
\begin{aligned}
\sum_{k=0}^{\infty} \operatorname{Pr} & \left(T_{x, 0}^{n}=k\right) z^{k} \\
& =\sum_{k=0}^{\infty} \operatorname{Pr}\left(T_{x, 0}^{n-1}=k\right) z^{k}+\sum_{k=0}^{\infty} \operatorname{Pr}\left(T_{x, n-1}^{0}+T_{n-1,0}^{n}=k\right) z^{k} \\
& =\sum_{k=0}^{\infty} \operatorname{Pr}\left(T_{x, 0}^{n-1}=k\right) z^{k}+\sum_{k=0}^{\infty} \sum_{l=0}^{k} \operatorname{Pr}\left(T_{x, n-1}^{0}=l\right) \operatorname{Pr}\left(T_{n-1,0}^{n}=k-l\right) z^{k} \\
& =\sum_{k=0}^{\infty} \operatorname{Pr}\left(T_{x, 0}^{n-1}=k\right) z^{k}+\sum_{k=0}^{\infty} \operatorname{Pr}\left(T_{x, n-1}^{0}=k\right) z^{k} \sum_{k=0}^{\infty} \operatorname{Pr}\left(T_{n-1,0}^{n}=k\right) z^{k} .
\end{aligned}
$$

In other words,

$$
C_{x, 0}^{n}(z)=C_{x, 0}^{n-1}(z)+C_{x, n-1}^{0}(z) C_{n-1,0}^{n}(z)
$$

For $1 \leq y \leq n-1$, every random path on $L$ from $x$ to $y$ without having passed any of the endpoints of $L, 0$, or $n$ can be regarded as a path from $x$ to $y$ generated by a random walk on $\mathbb{Z}_{n}=\{0, \ldots, n-1\}$ with a sink 0 and a probability distribution $\mu$ defined by $\mu(1)=\mu(-1)=1 / 2$. It is necessary to observe that a path on $L$ from $x$ to 0 without having hit $n-1$ is a path on $\mathbb{Z}_{n}$ from $x$ to 0 without having hit $n-1$. And a path on $L$ which hits $n-1$ before arriving at 0 is a path on $\mathbb{Z}_{n}$ which hits $n-1$ before arriving at 0 . For $0 \leq y, t \leq n-1$, let $\widetilde{T}_{x, y}$ be the first hitting time on $\mathbb{Z}_{n}$ from $x$ to $y$ and let $\tilde{T}_{x, y}^{t}$ be the first hitting time on $\mathbb{Z}_{n}$ from $x$ to $y$ without having hit $t$. Equation (2.14) implies that for $1 \leq x \leq n-2$,

$$
\begin{aligned}
C_{x, 0}^{n-1}(z) & =\sum_{k=0}^{\infty} \operatorname{Pr}\left(\tilde{T}_{x, 0}^{n-1}=k\right) z^{k}=V_{n}(x, 0, n-1, z), \\
C_{x, n-1}^{0}(z) & =\sum_{k=0}^{\infty} \operatorname{Pr}\left(\tilde{T}_{x, n-1}^{0}=k\right) z^{k}=V_{n}(x, n-1,0, z) .
\end{aligned}
$$

Equations (2.19) and (2.20) imply that for $1 \leq x \leq n-2$,

$$
C_{x, 0}^{n}(z)=V_{n}(x, 0, n-1, z)+V(x, n-1,0, z) C_{n-1,0}^{n}(z)
$$


Equations (2.17) and (2.21) imply that

$$
C_{n-1,0}^{n}(z)=\frac{z V_{n}(n-2,0, n-1, z)}{2-z V_{n}(n-2, n-1,0, z)}=\frac{z f(z)}{2+z g(z)},
$$

and for $1 \leq x \leq n-2$,

$$
C_{x, 0}^{n}(z)=V_{n}(x, 0, n-1, z)+\frac{z V_{n}(x, n-1,0, z) f(z)}{2+z g(z)} .
$$

In particular,

$$
C_{1,0}^{n}(z)=-g(z)+\frac{z f(z)^{2}}{2+z g(z)}
$$

REMARK 2.4. Note that in the above proof we also have derived the generating function $C_{x, 0}^{n}(z)$ of the first hitting time from $x$ to 0 without having hit $n$ on $L$ and the symmetry implies $C_{x, n}^{0}(z)=C_{n-x, 0}^{n}(z)$.

3. On graphs with congestion points. In this section, we consider a simple random walk on a lollipop and a dumbbell, which obviously have cutvertices. A cutvertex is a vertex such that the graph without this vertex is not connected.

Those cutvertices represent typical congestion points which cause delays in various traffic systems such as computer networks, telecommunication, and the transportation systems. We are interested in the first hitting time which can be interpreted as the performance time of such models. Since they have relatively simple structures, the probability generating functions can be derived using basic probabilistic argument. But it is interesting as well to apply group representation technique for these models since they can be decomposed into several cyclic groups $\mathbb{Z}_{n}$. First we define the functions

$$
\begin{aligned}
& \phi(z)=\frac{1+(1-z) \sum_{k=1}^{2 n-1} \cos (\pi k)[1-\cos (\pi k / n) z]^{-1}}{1+(1-z) \sum_{k=1}^{2 n-1}[1-\cos (\pi k / n) z]^{-1}} \\
& \varphi(z)=\frac{1+(1-z) \sum_{k=1}^{2 n-1} \cos (\pi k / n)[1-\cos (\pi k / n) z]^{-1}}{1+(1-z) \sum_{k=1}^{2 n-1}[1-\cos (\pi k / n) z]^{-1}} \\
& \xi(z)=(3-2 z \varphi(z)+z g(z))(2+z g(z))-z^{2} f(z)^{2}, \\
& \psi(z)=6+5 z g(z)-2 z^{2} f(z)^{2} \\
& \eta(z)=z^{2} f(z)(2+z g(z))(2 z \varphi(z)-3),
\end{aligned}
$$

where $f(z)$ and $g(z)$ were defined by (2.15). 


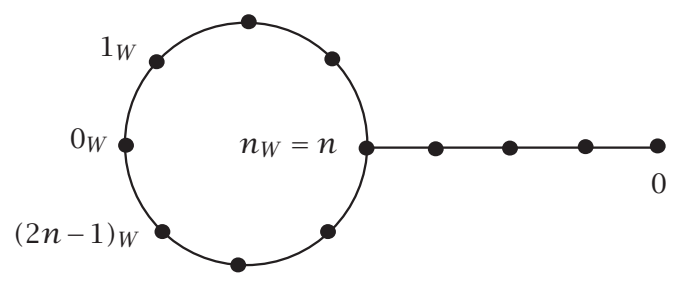

FIgURE 3.1. The lollipop $P$ in Theorem 3.1.

THEOREM 3.1. Consider a simple random walk on the lollipop $P$, which consists of a circle with $2 n$ vertices and a line segment with $n+1$ vertices, as in Figure 3.1. The generating function of the first hitting time from $0_{W}$ to 0 on the lollipop $P$ is given by

$$
F_{0_{W}, 0}(z)=\frac{z^{2} \phi(z) f(z)}{(3-2 z \varphi(z)+z g(z))(2+z g(z))-z^{2} f(z)^{2}} .
$$

Proof. Let $T_{x, y}$ be the first hitting time from $x$ to $y$ and let $T_{x, y}^{t}$ be the first hitting time from $x$ to $y$ without having hit $t$ on $P$. Let $F_{x, y}(z)=\sum_{k=0}^{\infty} \operatorname{Pr}\left(T_{x, y}=\right.$ k) $z^{k}$ and $F_{x, y}^{t}(z)=\sum_{k=0}^{\infty} \operatorname{Pr}\left(T_{x, y}^{t}=k\right) z^{k}$.

Note that each path from $0_{W}$ to 0 should pass through $n_{W}=n$ before arriving at 0 and restart from $n$ to 0 ; hence

$$
F_{0_{W}, 0}(z)=F_{0_{W}, n_{W}}(z) F_{n, 0}(z)
$$

Since each path from $0_{W}$ to $n_{W}$ can be regarded as the path from 0 to $n$ on $\mathbb{Z}_{2 n}$ with a probability distribution $\mu$ defined by $\mu(1)=\mu(-1)=1 / 2$, (2.13) implies that

$$
F_{0_{W}, n_{W}}(z)=U_{2 n}(0, n, z)=\phi(z) .
$$

Hence, we obtain

$$
F_{0_{W}, 0}(z)=\phi(z) F_{n, 0}(z)
$$

Note that each path from $n$ to 0 moves the first step to one of three directions: $(n+1)_{W},(n-1)_{W}$, and $n-1$, with probability $1 / 3$. We have

$$
3 F_{n, 0}(z)=z\left(F_{(n+1)_{W}, 0}(z)+F_{(n-1)_{W}, 0}(z)+F_{n-1,0}(z)\right) .
$$




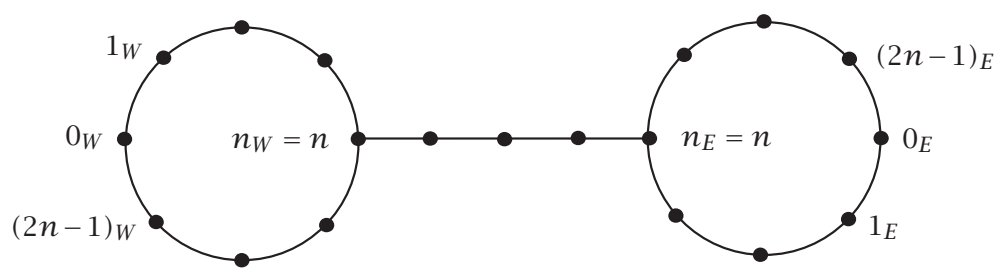

Figure 3.2. The dumbbell $Q$ in Theorem 3.2.

Similar to (3.3) and (3.4), we obtain

$$
\begin{aligned}
F_{(n+1)_{W}, 0}(z) & =F_{(n+1)_{W}, n_{W}}(z) F_{n, 0}(z), \\
F_{(n+1)_{W}, n_{W}}(z) & =U_{2 n}(n+1, n, z)=\varphi(z), \\
F_{(n-1)_{W}, 0}(z) & =F_{(n-1)_{W}, n_{W}}(z) F_{n, 0}(z), \\
F_{(n-1)_{W}, n_{W}}(z) & =U_{2 n}(n-1, n, z)=\varphi(z) .
\end{aligned}
$$

Hence, we have

$$
F_{(n+1)_{W}, 0}(z)=F_{(n-1)_{W}, 0}(z)=\varphi(z) F_{n, 0}(z) .
$$

On the other hand, each path from $n-1$ to 0 either arrives at 0 without having hit $n$ or hits $n$ before arriving at 0 , and then restarts random walk to reach 0 from $n=n_{W}$, and that the paths on $P$ from $n-1$ to 0 without having hit $n$ and from $n-1$ to $n$ without having hit 0 can be regarded as the paths on $L$ in Lemma 2.3. Hence, we have

$$
\begin{aligned}
F_{n-1,0}(z) & =F_{n-1,0}^{n}(z)+F_{n-1, n}^{0}(z) F_{n_{W}, 0}(z) \\
& =C_{n-1,0}^{n}(z)+C_{n-1, n}^{0}(z) F_{n, 0}(z) .
\end{aligned}
$$

Equations (3.6), (3.8), and (3.9) and Lemma 2.3(i) imply

$$
\begin{aligned}
F_{n, 0}(z) & =\frac{z C_{n-1,0}^{n}(z)}{3-2 z \varphi(z)-z C_{n-1, n}^{0}(z)} \\
& =\frac{z^{2} f(z)}{(3-2 z \varphi(z)+z g(z))(2+z g(z))-z^{2} f(z)^{2}},
\end{aligned}
$$

which, together with (3.5), implies the theorem.

THEOREM 3.2. Consider a simple random walk on the dumbbell $Q$, which consist of a line segment with $n+1$ vertices and two circles each with $2 n$ vertices, as in Figure 3.2. The generating function of the first hitting time from $0_{W}$ to $0_{E}$ on the dumbbell $Q$ is given by

$$
\widetilde{F}_{0_{W}, 0_{E}}(z)=\frac{2 z^{4} \phi(z) f(z)^{2}}{\psi(z) \xi(z)-\eta(z)} .
$$


Proof. Let $\tilde{T}_{x, y}$ be the first hitting time from $x$ to $y$ and let $\tilde{T}_{x, y}^{t}$ be the first hitting time from $x$ to $y$ without having hit $t$ on $Q$. Let $\widetilde{F}_{x, y}(z)=\sum_{k=0}^{\infty} \operatorname{Pr}\left(\tilde{T}_{x, y}=\right.$ $k) z^{k}$ and $\tilde{F}_{x, y}^{t}(z)=\sum_{k=0}^{\infty} \operatorname{Pr}\left(\tilde{T}_{x, y}^{t}=k\right) z^{k}$.

Note that each path from $0_{W}$ to $0_{E}$ should pass through $n_{E}$ before arriving at $0_{E}$ and restart a random walk from $n_{E}$ to $0_{E}$; we have

$$
\widetilde{F}_{0_{W}, 0_{E}}(z)=\widetilde{F}_{0_{W}, n_{E}}(z) \widetilde{F}_{n_{E}, 0_{E}}(z)
$$

Since each path from $0_{W}$ to $n_{E}$ on the dumbell $Q$ can be regarded as a path from $0_{W}$ to 0 on the lollipop $P$, Theorem 3.1 implies

$$
\widetilde{F}_{0_{W}, n_{E}}(z)=F_{0_{W}, 0}(z)=\frac{z^{2} \phi(z) f(z)}{(3-2 z \varphi(z)+z g(z))(2+z g(z))-z^{2} f(z)^{2}} .
$$

Note that a path starting from $n_{E}$ and ending at $0_{E}$ moves the first step randomly to one of three directions: $(n-1)_{E},(n+1)_{E}$, and 1 , with probability $1 / 3$. In other words,

$$
3 \widetilde{F}_{n_{E}, 0_{E}}(z)=z\left(\tilde{F}_{(n-1)_{E}, 0_{E}}(z)+\tilde{F}_{(n+1)_{E}, 0_{E}}(z)+\widetilde{F}_{1,0_{E}}(z)\right)
$$

By symmetry,

$$
\widetilde{F}_{(n-1)_{E}, 0_{E}}(z)=\widetilde{F}_{(n+1)_{E}, 0_{E}}(z)
$$

Since each random path from $(n-1)_{E}$ to $0_{E}$ either arrives at $0_{E}$ without having hit $n_{E}$ or hits $n_{E}$ before arriving at $0_{E}$ and then restarts random walk to reach $0_{E}$ from $n_{E}$, we have

$$
\widetilde{F}_{(n-1)_{E}, 0_{E}}(z)=\widetilde{F}_{(n-1)_{E}, 0_{E}}^{n_{E}}(z)+\widetilde{F}_{(n-1)_{E}, n_{E}}^{0_{E}}(z) \widetilde{F}_{n_{E}, 0_{E}}(z) .
$$

Note that each path from $(n-1)_{E}$ to $0_{E}$ without having hit $n_{E}$ on $Q$ can be regarded as a path from $n-1$ to 0 on $L$ in Lemma 2.3;

$$
\tilde{F}_{(n-1)_{E}, 0_{E}}^{n_{n_{E}}}(z)=C_{n-1,0}^{n}(z)=\frac{z f(z)}{2+z g(z)} .
$$

Similarly, we have

$$
\widetilde{F}_{(n-1)_{E}, n_{E}}^{0_{E}}(z)=C_{n-1, n}^{0}(z)=-g(z)+\frac{z f(z)^{2}}{2+z g(z)} .
$$

Hence, we have

$$
\begin{aligned}
\widetilde{F}_{(n-1)_{E}, 0_{E}}(z) & =\widetilde{F}_{(n+1)_{E}, 0_{E}}(z) \\
& =\frac{z f(z)}{2+z g(z)}+\left(-g(z)+\frac{z f(z)^{2}}{2+z g(z)}\right) \widetilde{F}_{n_{E}, 0_{E}}(z) .
\end{aligned}
$$


On the other hand, each path from 1 to $0_{E}$ on $Q$ should pass through 0 and restart from $0=n_{E}$ to $0_{E}$; we have

$$
\tilde{F}_{1,0_{E}}(z)=\tilde{F}_{1,0}(z) \tilde{F}_{n_{E}, 0_{E}}(z) .
$$

Since each path from 1 to 0 either arrives at 0 without having hit $n$ or hits $n$ and then restarts from $n$ to 0 ,

$$
\tilde{F}_{1,0}(z)=\widetilde{F}_{1,0}^{n}(z)+\widetilde{F}_{1, n}^{0}(z) \widetilde{F}_{n, 0}(z)
$$

But those paths on $Q$ from 1 to 0 without having hit $n$ and from 1 to $n$ without having hit 0 can be regarded as those paths on $L$ in Lemma 2.3, that is,

$$
\begin{aligned}
& \tilde{F}_{1,0}^{n}(z)=C_{1,0}^{n}(z)=\frac{z f(z)}{2+z g(z)}, \\
& \tilde{F}_{1, n}^{0}(z)=C_{1, n}^{0}(z)=-g(z)+\frac{z f(z)^{2}}{2+z g(z)} .
\end{aligned}
$$

Note that each path from $n$ to 0 on $Q$ can be regarded as the path from $n$ to 0 on $P$ and formula (3.10) implies

$$
\widetilde{F}_{n, 0}(z)=F_{n, 0}(z)=\frac{z^{2} f(z)}{(3-2 z \varphi(z)+z g(z))(2+z g(z))-z^{2} f(z)^{2}} .
$$

Hence, we have

$$
\tilde{F}_{1,0}(z)=\frac{z f(z)(3-2 z \varphi(z))}{(3-2 z \varphi(z)+z g(z))(2+z g(z))-z^{2} f(z)^{2}} .
$$

Formulas (3.20) and (3.24) imply

$$
\tilde{F}_{1,0_{E}}(z)=\left(\frac{z f(z)(3-2 z \varphi(z))}{(3-2 z \varphi(z)+z g(z))(2+z g(z))-z^{2} f(z)^{2}}\right) \tilde{F}_{n_{E}, 0_{E}}(z),
$$

and hence (3.14), (3.19), and (3.25) imply the theorem.

4. Conclusions. It is not hard to modify our argument to derive the probability generating functions on necklace graphs which consist of several circles and some lines, and also on more general finite graphs which consist of several Cayley graphs. The probabilistic algorithms of random walks on such graphs may give rich information on probabilistic properties of several combinatorial models, such as networks and traffic congestion problems, in terms of hitting times.

Since we have derived the explicit formulas of the probability generating functions in the previous sections, we can efficiently compute the distribution of the first hitting time; namely, the probability, the average, variance, 


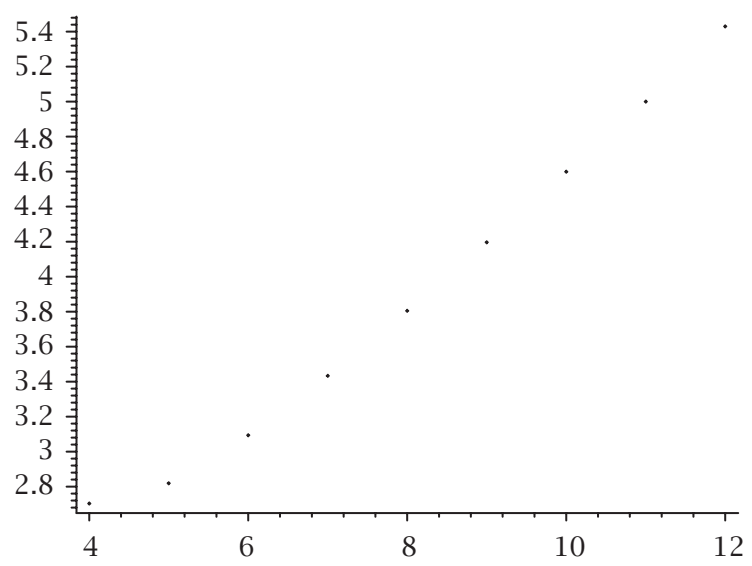

FIGURE 4.1. The expectations computed by Theorem 3.1 and Maple.

and higher-order moments using Taylor series expansion (see, e.g., [16]). Let $p_{k}$ denote the probability of the first hitting time from $x$ to $y$ being $k$. The probability generating function, defined by

$$
A(z)=\sum_{k=0}^{\infty} p_{k} z^{k}
$$

gives the value $p_{k}$ as

$$
p_{k}=\frac{1}{k !} A^{(k)}(0)
$$

where $A^{(k)}(0)$ denotes the $k$ th derivative of $A(z)$ at $z=0$. Moreover, we can compute the average, variance, and higher-order moments using higher derivatives, for example,

$$
\mathrm{E}(T)=\sum_{k} k p_{k}=A^{\prime}(1), \quad \operatorname{Var}(T)=A^{\prime \prime}(1)+A^{\prime}(1)-A^{\prime}(1)^{2} .
$$

Some computational softwares, such as Maple or Mathematica, allow us to compute them very quickly with high accuracy. For example, using Maple and Theorem 3.1, the expectations of the first hitting time on the lollipop have been computed up to $n=12$ as shown in Figure 4.1.

ACKNOWLEDGments. I would like to thank the referee for careful reading of the paper and several helpful comments. This research was supported by the Deutsche Forschungsgemeinschaft through the European Graduate Program “Combinatorics, Geometry, and Computation,” no. GRK 588/1. 


\section{REFERENCES}

[1] D. Aldous, Hitting times for random walks on vertex-transitive graphs, Math. Proc. Cambridge Philos. Soc. 106 (1989), no. 1, 179-191.

[2] D. Aldous and J. Fill, Reversible Markov chains and random walks on graphs, in preparation.

[3] B. Bollobás, Modern Graph Theory, Graduate Texts in Mathematics, vol. 184, Springer-Verlag, New York, 1998.

[4] D. Coppersmith, P. Doyle, P. Raghavan, and M. Snir, Random walks on weighted graphs and applications to on-line algorithms, J. Assoc. Comput. Mach. 40 (1993), no. 3, 421-453.

[5] P. Diaconis, Group Representations in Probability and Statistics, Institute of Mathematical Statistics Lecture Notes-Monograph Series, vol. 11, Institute of Mathematical Statistics, California, 1988.

[6] P. Diaconis and L. Saloff-Coste, Comparison theorems for reversible Markov chains, Ann. Appl. Probab. 3 (1993), no. 3, 696-730.

[7] P. Diaconis and D. Stroock, Geometric bounds for eigenvalues of Markov chains, Ann. Appl. Probab. 1 (1991), no. 1, 36-61.

[8] P. G. Doyle and J. L. Snell, Random Walks and Electric Networks, Carus Mathematical Monographs, vol. 22, Mathematical Association of America, District of Columbia, 1984.

[9] B. González-Arévalo and J. L. Palacios, Expected hitting times for random walks on weak products of graphs, Statist. Probab. Lett. 43 (1999), no. 1, 33-39.

[10] M. Jerrum, Mathematical foundations of the Markov chain Monte Carlo method, Probabilistic Methods for Algorithmic Discrete Mathematics (M. Habib, C. McDiarmid, J. Ramirez-Alfonsin, and B. Reed, eds.), Algorithms Combin., vol. 16, Springer, Berlin, 1998, pp. 116-165.

[11] R. Motwani and P. Raghavan, Randomized Algorithms, Cambridge University Press, Cambridge, 1995.

[12] J. R. Norris, Markov Chains, Cambridge Series in Statistical and Probabilistic Mathematics, Cambridge University Press, Cambridge, 1998.

[13] J. L. Palacios and P. Tetali, A note on expected hitting times for birth and death chains, Statist. Probab. Lett. 30 (1996), no. 2, 119-125.

[14] P. Tetali, Random walks and the effective resistance of networks, J. Theoret. Probab. 4 (1991), no. 1, 101-109.

[15]_ _ _ Design of on-line algorithms using hitting times, SIAM J. Comput. 28 (1999), no. 4, 1232-1246.

[16] H. S. Wilf, Generatingfunctionology, 2nd ed., Academic Press, Massachusetts, 1994.

Mihyun Kang: Department of Computer Science, Humboldt University Berlin, Unter den Linden 6, 10099 Berlin, Germany

E-mail address: kang@informatik.hu-berlin.de 


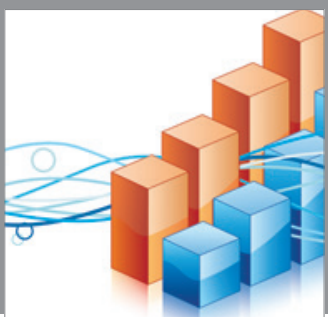

Advances in

Operations Research

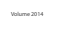

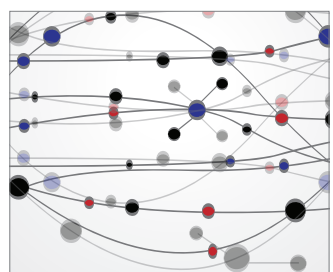

\section{The Scientific} World Journal
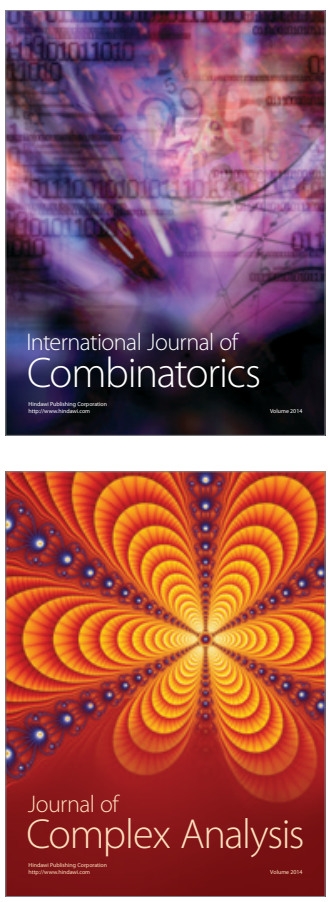

International Journal of

Mathematics and

Mathematical

Sciences
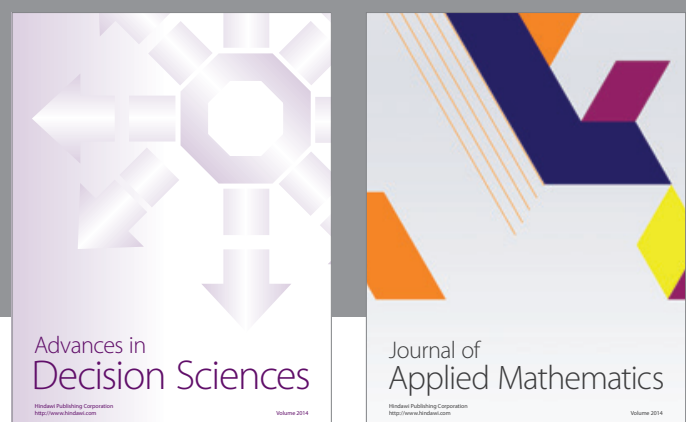

Journal of

Applied Mathematics
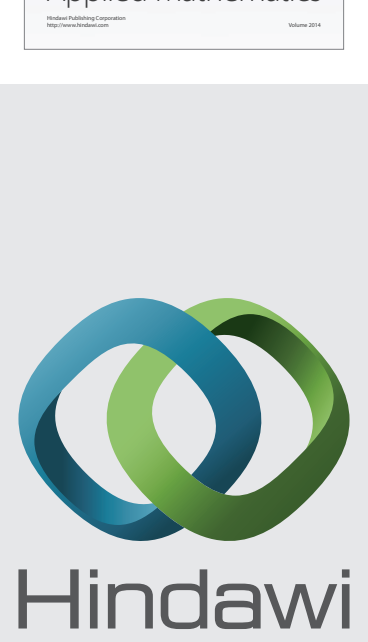

Submit your manuscripts at http://www.hindawi.com
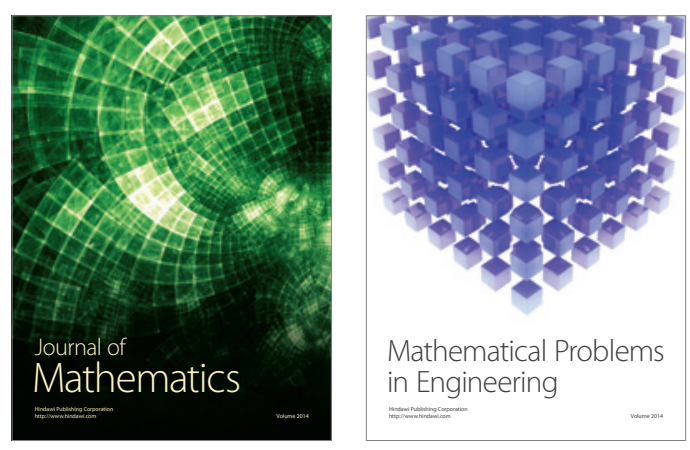

Mathematical Problems in Engineering
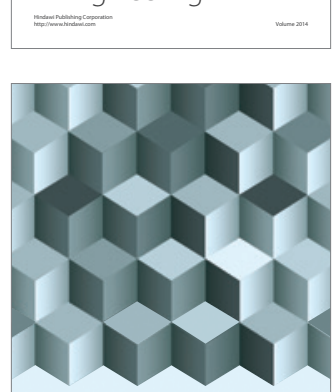

Journal of

Function Spaces
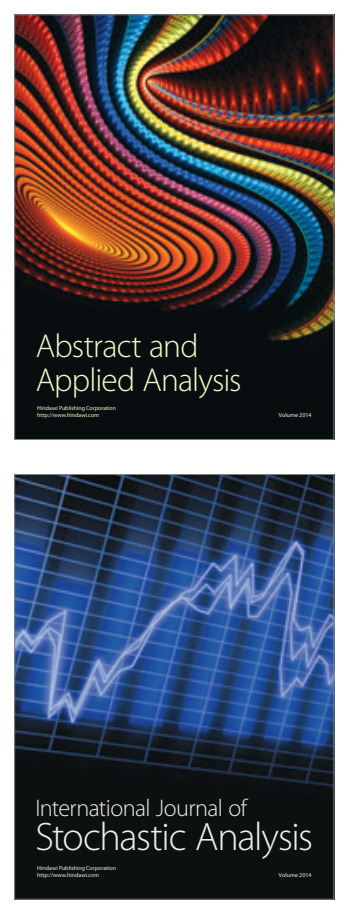

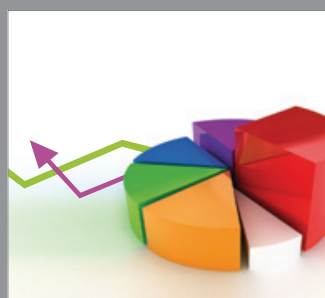

ournal of

Probability and Statistics

Promensencen
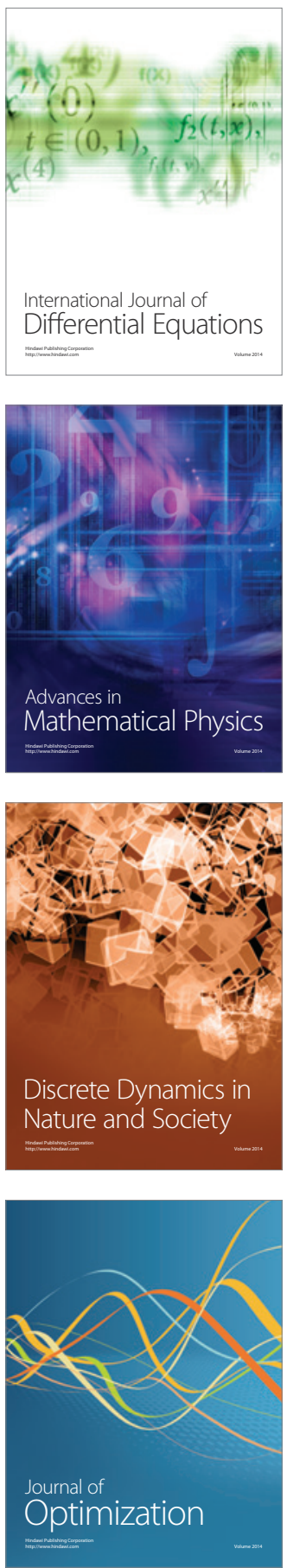